\title{
Corticosteróide Antenatal: Ciclo Único versus Múltiplo - Comparação de Resultados
}

Antenatal Corticosteroids: Single versus Multiple Courses - Comparison of the Results

Joice Fabiola Meneguel, Ruth Guinsburg, Milton Harumi Miyoshi, Clovis de Araújo Peres, Benjamin Israel Kopelman, Luiz Camano

\begin{abstract}
RESUMO
Objetivo: avaliar se ciclos múltiplos de corticosteróide antenatal (CEA) são mais efetivos, em relação ao ciclo único, na redução da morbimortalidade de prematuros.

Métodos: estudo retrospectivo envolvendo 184 neonatos com idade gestacional inferior a 34 semanas, nascidos em hospital de nível terciário de São Paulo no período de janeiro de 1988 a dezembro de 1998. Os pacientes foram divididos conforme o tratamento em: ciclo único (n=135): se a gestante recebeu um ciclo completo do CEA (2 doses de betametasona ou 4 doses de dexametasona, entre 24 horas e 7 dias do parto) e ciclos múltiplos (n=49): se a gestante foi tratada com dois ou mais ciclos completos. Foram comparados entre os dois grupos do estudo: síndrome do desconforto respiratório (SDR), mortalidade intra-hospitalar e morbidade neonatal combinada (ocorrência de um ou mais: SDR, hemorragia periintraventricular, displasia broncopulmonar, enterocolite necrosante, sepse ou óbito intrahospitalar). Os dados maternos e neonatais foram comparados pelo teste t de Student ou Mann-Whitney para as variáveis numéricas e pelo $\chi^{2}$ ou exato de Fisher, com cálculo das "odds ratio" e seu intervalo de confiança, para as variáveis categóricas.

Resultados: não foi demonstrada diferença significante na evolução dos neonatos do grupo ciclo único em relação ao múltiplo quanto à ocorrência de SDR (ciclo único: $22 \%$ e ciclo múltiplo: 18\%), mortalidade intra-hospitalar (único 18\% e múltiplo 12\%) e morbidade neonatal combinada (único $62 \%$ e múltiplo 63\%).

Conclusões: o uso de ciclos múltiplos de CEA não diminuiu a morbimortalidade de prematuros. Esse estudo reforça a recomendação atual da utilização de ciclo único de corticosteróide para a maturação fetal em gestantes em trabalho de parto prematuro.
\end{abstract}

PALAVRAS-CHAVE: Corticosteróide antenatal. Recém-nascido prematuro. Maturação fetal.

\section{Introdução}

A utilização do corticosteróide antenatal para a maturação pulmonar fetal iniciou-se em 1972, após a pesquisa pioneira de Liggins e Howie ${ }^{1}$. Estudos realizados posteriormente demonstraram os benefícios do corticosteróide antenatal na redução da ocorrência, em prematuros, da síndrome

Disciplina de Pediatria Neonatal do Departamento de Pediatria da Universidade Federal de São Paulo/ Escola Paulista de Medicina

Correspondência: Joice Fabíola Meneguel

Rua Maurício Jacquey, 421

09635-080 - São Bernardo do Campo - SP

Telefone: 5579-1676

e-mail:dpn@osite.com.br do desconforto respiratório, da hemorragia peri e intraventricular e da mortalidade neonatal ${ }^{2}$. Os efeitos benéficos dos corticosteróides se estendem até sete a dez dias após a aplicação da dose inicial e, após 14 a 21 dias, os efeitos benéficos da corticoterapia tendem a desaparecer ${ }^{1}$. Com base nessas observações, muitos obstetras começaram a utilizar ciclos semanais repetidos do corticosteróide antenatal. No entanto, a preocupação com o uso disseminado de ciclos múltiplos do corticosteróide é crescente, porque não há evidências das vantagens dos ciclos múltiplos sobre o ciclo único e existem temores de potencialização de possiveis efeitos adversos ${ }^{3}$.

Se por um lado a utilização de doses repeti- 
das de corticosteróides leva a melhoria progressiva na função pulmonar, demonstrada em experimentos com animais ${ }^{4}$, há efeitos deletérios de ciclos múltiplos de corticosteróides na função do eixo hipotalâmico-hipofisário-adrenal ${ }^{5}$, no peso e no volume cerebral ${ }^{6}$ e no peso de nascimento ${ }^{7}$, também demonstrados em estudos animais. Alguns estudos envolvendo seres humanos, todos retrospectivos, demonstraram associação entre ciclos múltiplos de corticosteróide antenatal e redução do peso de nascimento ${ }^{8,9}$, do perímetro cefálico ${ }^{8}$, maior risco de óbito ${ }^{9}$ e infecção neonatal ${ }^{10}$ e supressão adrenal mais prolongada e evidente ${ }^{9}$.

Devido às dúvidas ainda existentes quanto ao efeito dos múltiplos ciclos de corticosteróide antenatal, um grande estudo ${ }^{11}$ prospectivo, randomizado e duplo-cego foi iniciado em 1996 em 13 centros universitários norte-americanos, envolvendo a utilização de placebo ou ciclos repetidos de corticosteróides, em gestantes com ameaça de parto prematuro, após a utilização de um ciclo inicial do esteróide. Os ciclos do esteróide ou do placebo foram repetidos até a $34^{\mathrm{a}}$ semana de gestação ou até a resolução do parto. Esse estudo deveria, inicialmente, envolver 1000 gestantes. No entanto, após a verificação dos resultados em 502 pacientes, os autores optaram pela interrupção da pesquisa. Os ciclos múltiplos não se mostraram efetivos na redução da morbidade neonatal combinada, que incluiu a ocorrência de sindrome do desconforto respiratório, displasia broncopulmonar, hemorragia periintraventricular, sepse comprovada, enterocolite necrosante e óbito perinatal (ciclos múltiplos: $22,5 \%$ e ciclo único: $28 \%$; RR 0,80; IC 95\% : 0,59-1,10).

Em um momento de restrição aos ciclos múltiplos do corticosteróide antenatal, decidimos realizar esse levantamento retrospectivo envolvendo neonatos expostos a ciclos múltiplos ou único, para avaliar se os ciclos múltiplos de corticosteróide antenatal foram mais efetivos na redução da morbimortalidade de prematuros nascidos em uma instituição universitária terciária de São Paulo.

\section{Pacientes e Métodos}

O presente estudo constitui uma coorte retrospectiva baseada na análise dos prontuários de pacientes com idade gestacional inferior a 34 semanas e de seus respectivos filhos, nascidos no período de janeiro de 1988 a dezembro de 1998, no Hospital São Paulo, Universidade Federal de São Paulo. O protocolo de pesquisa foi aprovado pela Comissão de Ética em Pesquisa da instituição.

Os pacientes foram divididos em dois grupos: ciclo único $(n=135)$ - recém-nascidos cujas mães foram expostas a um ciclo completo do corticosteróide antenatal, constituído de 2 doses de betametasona ou 4 doses de dexametasona entre 24 horas e 7 dias do parto, e ciclo múltiplo $(n=49)$ recém-nascidos cujas mães foram expostas a dois ou mais ciclos completos do corticosteróide antenatal. A prescrição do número de doses de corticosteróide foi baseada na decisão individual do obstetra que acompanhava a paciente.

Os critérios de exclusão da população estudada foram: presença de anomalias congênitas maiores e emprego do corticosteróide pela gestante para outra finalidade que não a indução da maturação fetal.

Os prontuários maternos e dos recém-nascidos foram pesquisados para a obtenção das seguintes informações demográficas maternas: idade e raça; doenças clínicas preexistentes; patologias obstétricas na gestação atual; realização de acompanhamento pré-natal ( $>4$ consultas); necessidade de tocolíticos; presença de amniorrexe prolongada, definida como rotura das membranas amnióticas por tempo superior a 24 horas; presença de corioamnionite; tempo decorrido desde a internação até o parto e tipo de parto. Ainda dos prontuários maternos foram obtidos dados a respeito da prescrição de corticosteróides. Foram analisadas quais as drogas utilizadas (betametasona ou dexametasona), o número de doses aplicadas, o número de horas ou dias entre a aplicação da primeira dose e o momento do parto e o número de ciclos recebidos pela gestante.

Dentre os dados coletados dos prontuários dos recém-nascidos destacaram-se: necessidade de reanimação em sala de parto; índice de Apgar no primeiro e no quinto minuto de vida; sexo; peso ao nascimento; idade gestacional determinada pela data da última menstruação materna, por dados ultra-sonográficos ou pelo exame neonatal ${ }^{12,13}$; classificação do recém-nascido ${ }^{14}$ e presença de gemelaridade.

A evolução clínica dos recém-nascidos na Unidade Neonatal foi avaliada para detectar a ocorrência dos principais desfechos clínicos: sindrome do desconforto respiratório (definida quando o recém-nascido apresentou desconforto respiratório de início precoce, até seis horas de vida, e de evolução progressiva nas primeiras 24 horas, com quadro radiológico compatível), óbito intra-hospitalar (avaliou-se a idade de ocorrência do óbito e a sua causa imediata relatada pelo neonatologista que atendeu o paciente ou pelo patologista que realizou a necrópsia), uso de surfactante (dentre os pacientes com diagnóstico de síndrome do desconforto respiratório, foi verificado o número de doses e o tipo de surfactante exógeno utilizado, com a ressalva de que o surfactante exógeno vinha sendo utilizado na unidade em questão de 
maneira regular desde fevereiro de 1992), ventilação mecânica (foi avaliada a necessidade e a duração da ventilação mecânica), displasia broncopulmonar (definida como dependência de oxigênio aos 28 dias de vida, com alterações radiológicas fixas), hemorragia periintraventricular (por ultra-sonografia transfontanelar à beira do leito, geralmente no quarto, décimo e trigésimo dias de vida. O grau da hemorragia foi definido segundo a classificação de Papile et al. ${ }^{15}$, enterocolite necrosante (presença ou ausência do diagnóstico clínico-radiológico ou cirúrgico de enterocolite necrosante, com estadiamento segundo Walsh \& Kliegman ${ }^{16}$ ), sepse comprovada (presença de hipo ou hipertermia e de pelo menos um dos seguintes sinais ou sintomas: taquicardia, bradicardia e/ou hipoperfusão periférica, apnéia, taquipnéia e/ou desconforto respiratório e alterações na contagem total de leucócitos, acompanhada de hemocultura positiva) e, finalmente, a morbidade neonatal combinada (definida como a presença de qualquer um dos seguintes desfechos: sindrome do desconforto respiratório, hemorragia periintraventricular, displasia broncopulmonar, enterocolite necrosante, sepse comprovada ou óbito intra-hospitalar ${ }^{11,17}$ ).

Para a análise estatística foram aplicados os testes do $\chi^{2}{ }^{18}$ ou exato de Fisher ${ }^{18}$ para a comparação das variáveis categóricas entre os pacientes expostos aos ciclos múltiplos ou único do corticosteróide antenatal. O teste $t$ de Student ${ }^{19}$ foi utilizado para a comparação das variáveis numéricas. O tempo de internação hospitalar, a duração da ventilação mecânica e o número de doses de surfactante, por não apresentarem distribuição normal, foram comparados entre os grupos com o teste de Mann-Whitney ${ }^{20}$. A comparação dos desfechos clínicos dos dois grupos de estudo foi realizada com o cálculo da "odds ratio" e de seu intervalo de confiança ${ }^{18}$. Em todos os testes fixou-se o nível de significância em $0,05(\alpha \leq 5 \%)$.

\section{Resultados}

No período do estudo, segundo os dados obtidos dos registros dos recém-nascidos atendidos na Unidade Neonatal do Hospital São Paulo/UNIFESPEPM, nasceram 1051 prematuros com menos de 34 semanas de idade gestacional. Destes 1051 pacientes, foi possivel a revisão de 1023 (99,5\%) prontuários maternos e observou-se que 258 mães fizeram uso de corticosteróide para induzir a maturidade fetal até sete dias antes da resolução do parto e, destes, 244 (95\%) prontuários dos respectivos recém-nascidos foram recuperados. Dentre as 244 pacientes que receberam o corticosteróide antenatal foram excluídas aquelas que receberam ciclos incompletos do corticosteróide. Desse modo, no presente estudo foram incluídos os 184 pacientes que receberam ciclos completos do corticosteróide, divididos entre 135 (73\%) com ciclo único e $49(27 \%)$ com ciclos múltiplos. As gestantes expostas a ciclos múltiplos de corticosteróides receberam em média 2,7 ciclos por paciente, variando de dois a sete ciclos.

Em relação aos dados maternos, observa-se na Tabela 1 maior freqüência de acompanhamento pré-natal (múltiplo $77 \%$ e único $41 \%$ ) e parto cesáreo (múltiplo 80\% e único 60\%) e tempo de internação mais prolongado (múltiplo 16 dias e único 6 dias) dentre as gestantes que receberam ciclos múltiplos da medicação. Não houve diferenças entre os dois grupos de estudo quanto à raça, idade materna, presença de diabetes, uso de tocolíticos, amniorrexe por tempo superior a 24 horas e ocorrência de corioamnionite. Pode-se notar, entretanto, uma incidência mais elevada de moléstia hipertensiva específica da gravidez nas pacientes que receberam ciclo único do corticosteróide antenatal (múltiplo 12\% e único 26\%).

Tabela 1 - Características demográficas das mães e dos recém-nascidos que receberam ciclos único ou múltiplos de corticosteróide antenatal, expressas em número de pacientes (porcentagem) ou em média (desvio padrão).

$\begin{array}{cccc}\text { Ciclo único } & \text { Ciclo múltiplo } & \mathrm{p} \\ (\mathrm{n}=135) & (\mathrm{n}=49) & \\ \mathrm{n} \quad \% & \mathrm{n} \quad \% & \end{array}$

\begin{tabular}{|c|c|c|c|c|c|}
\hline \multicolumn{6}{|l|}{ Características maternas } \\
\hline Idade (média) & 27 & & 28 & & 0,321 ** \\
\hline Branca & 81 & 60 & 34 & 70 & $0,540^{*}$ \\
\hline Pré-natal presente & 56 & 41 & 38 & 77 & $<0,001^{\star}$ \\
\hline DHEG & 36 & 26 & 6 & 12 & $0,062^{\star}$ \\
\hline Diabetes & 6 & 4 & 2 & 4 & $1,000^{*}$ \\
\hline Tocolíticos & 28 & 20 & 10 & 20 & $0,875^{\star}$ \\
\hline Amniorrexe $>24 \mathrm{~h}$ & 30 & 22 & 13 & 26 & $0,679 *$ \\
\hline Corioamnionite & 7 & 5 & 4 & 8 & $0,486^{\star}$ \\
\hline Parto cesáreo & 82 & 60 & 39 & 80 & $0,027^{*}$ \\
\hline Internação em dias (média) & 6 & \multicolumn{2}{|r|}{16} & & $<0,001^{\star \star *}$ \\
\hline \multicolumn{6}{|l|}{ Características neonatais } \\
\hline Peso (g) & 1260 & 384 & 1305 & 389 & $0,488^{\star \star}$ \\
\hline Idade gestacional (sem) & 31 & 2 & 32 & 2 & $0,690 * *$ \\
\hline Sexo masculino & 77 & 52 & 24 & 49 & $0,421^{*}$ \\
\hline Reanimação & 125 & 92 & 43 & 87 & $0,374^{*}$ \\
\hline Apgar 1 & 6 & 2 & 6 & 3 & $0,707^{\star \star}$ \\
\hline Apgar 5 & 8 & 2 & 8 & 2 & $0,725^{\star *}$ \\
\hline PIG & 39 & 28 & 13 & 26 & $0,897^{*}$ \\
\hline Gemelares & 12 & $9^{\prime \prime}$ & 7 & 14 & $0,429^{*}$ \\
\hline
\end{tabular}


Quanto às características demográficas dos recém-nascidos, também expressas na Tabela 1 , evidencia-se que os neonatos expostos ao ciclo único ou múltiplo de corticosteróide antenatal apresentaram respectivamente, em média, 1260 a $1305 \mathrm{~g}$ de peso ao nascer e 31 e 32 semanas de idade gestacional, sendo o Apgar de 1 e 5 minutos, respectivamente, de 6 e 8 nos dois grupos. Notase ainda que os pacientes dos dois grupos eram similares no que diz respeito ao sexo, à necessidade de reanimação em sala de parto e à freqüência de recém-nascidos pequenos para a idade gestacional e de gemelares.

Em relação aos principais desfechos clínicos do presente estudo (Tabela 2), observa-se que, dos expostos ao ciclo único do corticosteróide antenatal, 30 (22\%) desenvolveram síndrome do desconforto respiratório, 25 (18\%) evoluíram para óbito intra-hospitalar e 84 (62\%) apresentaram uma ou mais entidades mórbidas sumarizadas no indice de morbidade neonatal combinada. Para aqueles neonatos expostos a ciclos múltiplos da medicação antenatal, a síndrome do desconforto respiratório ocorreu em 9 (18\%), o óbito em 6 (12\%) e a morbidade neonatal combinada em 31 (63\%). Ou seja, não houve diferença significante entre os grupos. Já em relação aos desfechos clínicos secundários, os resultados da Tabela 2 indicam que não ocorreu diminuição na necessidade de terapêutica com reposição de surfactante exógeno, no número de doses de surfactante utilizadas, na incidência de displasia broncopulmonar, hemorragia periintraventricular, enterocolite necrosante e sepse neonatal comprovada nos pacientes expostos a ciclos múltiplos do esteróide no período antenatal, comparados àqueles que receberam o ciclo único da droga. Houve redução, sem significância estatística, no número de dias de ventilação mecânica nos recém-nascidos aos quais foram administrados ciclos múltiplos do esteróide antenatal (ciclo único: 4 dias versus múltiplo: 2 dias; $p=0,08$ ), apesar de não ter se verificado nem mesmo tendência à redução da necessidade de ventilação mecânica nos neonatos expostos a ciclos múltiplos de corticosteróide antenatal.

Tabela 2 - Desfechos clínicos dos recém-nascidos que receberam ciclos único ou múltiplos de corticosteróide antenatal.

\begin{tabular}{|c|c|c|c|c|}
\hline & $\begin{array}{c}\text { Ciclo único } \\
\text { n (\%) }\end{array}$ & $\begin{array}{c}\text { Ciclo múltiplo } \\
\text { n (\%) }\end{array}$ & OR & IC95\% \\
\hline Síndrome do desconforto respiratório & $30(22)$ & $9(18)$ & 1,27 & $(0,52-3,17)$ \\
\hline Necessidade de ventilação & $75 \quad(55)$ & $23(47)$ & 1,41 & $(0,70-2,87)$ \\
\hline Necessidade de surfactante & 19 (14) & $4 \quad(8)$ & 1,84 & $(0,55-6,80)$ \\
\hline Displasia bronco-pulmonar & 18 (13) & $6 \quad(12)$ & 1,10 & $(0,38-3,34)$ \\
\hline Sepse neonatal comprovada & $14(10)$ & $5 \quad(10)$ & 1,02 & $(0,32-3,46)$ \\
\hline Enterocolite necrosante & $11 \quad(8)$ & $5 \quad(10)$ & 0,78 & $(0,23-2,75)$ \\
\hline Óbito intra-hospitalar & $25(18)$ & $6 \quad(12)$ & 1,63 & $(0,58-4,78)$ \\
\hline Morbidade neonatal combinada & $84 \quad(62)$ & $31 \quad(63)$ & 0,96 & $(0,46-1,98)$ \\
\hline
\end{tabular}

A comparação entre os grupos está expressa em "odds ratio" e intervalo de confiança de 95\% (OR: IC95\%)

\section{Discussão}

A definição de ciclo múltiplo não está clara na literatura, porém a última recomendação da reunião de consenso promovida pelos National Institutes of Health estabelece que somente um ciclo completo de corticosteróide deveria ser indicado nas gestantes com risco de parto prematuro $^{17}$. Daí porque optamos por utilizar a definição de ciclo múltiplo como a administração de dois ou mais ciclos de corticosteróide antenatal.

Os corticosteróides são desencadeadores da morte celular programada e, desse modo, levam à diminuição do tamanho dos órgãos ${ }^{21}$. Estudos animais confirmam a redução do peso ao nascer após doses repetidas de corticosteróide antenatal ${ }^{7}$.
Quando analisamos as características demográficas dos recém-nascidos da coorte estudada, não observamos alterações do peso de nascimento dos pacientes que receberam ciclos múltiplos, comparados àqueles expostos ao ciclo único de corticosteróide antenatal. Talvez o pequeno número de pacientes disponiveis para essa comparação tenha comprometido o resultado, ou seja, a amostra não teve poder estatístico suficiente para demonstrar alterações significantes entre os grupos. Ainda, talvez, o número de repetição dos ciclos antenatais de corticosteróides tenha sido relativamente pequeno $(2,7$ ciclos $)$, insuficiente para promover a diminuição do peso de nascimento dos neonatos. Na literatura, os resultados clínicos quanto ao comprometimento do crescimento de conceptos expostos a ciclos repetidos de corticos- 
teróide antenatal são controversos. Alguns estudos relatam a diminuição do peso de nascimento dos conceptos que receberam ciclos múltiplos de corticosteróide antenatal. French et al. ${ }^{8}$ encontraram redução de $9 \%$ no peso ao nascer dos pacientes que receberam mais de três ciclos de corticosteróide antenatal. Banks et al. ${ }^{9}$, com base nos dados de 710 neonatos prematuros entre a $25^{\mathrm{a}}$ e a $32^{\mathrm{a}}$ semana de idade gestacional e por meio de um modelo de regressão linear, estimaram que o peso de nascimento diminui em 39 gramas nos conceptos que recebem mais de um ciclo de corticosteróide antenatal. Em ambos os estudos compararam-se esses recém-nascidos àqueles de idade gestacional similar expostos a um único ciclo do esteróide. Quando os pacientes são expostos a mais de dois ciclos da medicação, a redução do peso de nascimento é de 80 gramas em relação àqueles que receberam o ciclo único do corticosteróide. Outras investigações divergem das anteriores como a de Pratt et al. ${ }^{22}$, que considerou múltiplo qualquer número de ciclo maior que um, e a de Abbasi et al. ${ }^{23}$, na qual ciclo múltiplo foi definido como a exposição a dois ou mais ciclos de corticosteróide antenatal. Em ambos os estudos comparou-se o peso ao nascer de neonatos expostos a ciclos múltiplos ou únicos de corticosteróide neonatal e não se demonstrou alteração do peso de nascimento. Talvez, além do número de ciclos, esteja implicada na redução do peso ao nascer a época da gestação em que ocorre a repetição da exposição do concepto ao corticosteróide.

Em relação aos desfechos estudados, observou-se tendência à redução do número de dias de ventilação mecânica nos recém-nascidos expostos a ciclos múltiplos do esteróide antenatal (ciclo único: 4 dias versus múltiplo: 2 dias; $\mathrm{p}=0,08$ ). Ikegami et al. ${ }^{4}$ demonstraram melhora progressiva da complacência pulmonar, do índice de eficiência ventilatória e do volume pulmonar após o uso de duas, três e quatro doses de corticosteróide antenatal em ovelhas prematuras. Em estudos $\operatorname{clínicos}^{22,23}$, foi demonstrada a diminuição da incidência de síndrome do desconforto respiratório e a redução da necessidade do uso de oxigênio em prematuros expostos a ciclos múltiplos de corticosteróide antenatal, comparados a pacientes que receberam ciclos únicos da medicação, mostrando o efeito aditivo da repetição de ciclos de corticosteróide na maturação pulmonar. No entanto, análise atual dos efeitos de ciclos múltiplos dos corticosteróides na mortalidade de prematuros entre 26 a 32 semanas $^{24}$ mostra que os pacientes expostos a mais de três ciclos de corticosteróides apresentam maior freqüência de falência respiratória grave, com necessidade de suporte respiratório elevado e ocorrência de óbito nas primeiras seis horas de vida. Os autores associaram essa insuficiência respiratória grave ao efeito dos ciclos repetidos dos esteróides no desenvolvimento pulmonar durante a gestação. Em ratos foi demons- trado que os corticosteróides, quando utilizados no período de formação dos alvéolos, podem levar à hipoplasia pulmonar, com risco aumentado de hipertensão pulmonar ${ }^{25}$.

Optamos por utilizar a morbidade neonatal combinada como um dos desfechos a serem comparados entre os grupos do estudo, uma vez que essa medida vem sendo utilizada em pesquisas clínicas. Acredita-se que os ciclos múltiplos podem diminuir a ocorrência de algumas doenças (síndrome do desconforto respiratório) e aumentar a de outras complicações (sepse, enterocolite necrosante e mortalidade neonatal). Desse modo, ao combinarmos as morbidades, poderíamos avaliar melhor o efeito geral dos ciclos múltiplos do corticosteróide antenatal ${ }^{11}$. No estudo prospectivo e randomizado realizado por Guinn et al. ${ }^{11}$ não foi observada diferença entre os grupos expostos a ciclos repetidos ou não de corticosteróides quanto à morbidade neonatal combinada. Em outro estudo retrospectivo ${ }^{26}$, muito semelhante ao aqui apresentado, 143 pacientes que receberam ciclo único foram comparados a 61 pacientes expostos a mais de um ciclo de corticosteróide antenatal. Os autores não encontraram diferenças entre os grupos em relação à morbidade neonatal combinada, incluindo síndrome do desconforto respiratório, hemorragia periintraventricular, sepse, persistência do canal arterial e enterocolite necrosante. Quando os pacientes que receberam três ou mais ciclos foram comparados com aqueles com menos ciclos, houve aumento da morbidade neonatal combinada $(29 \%$ vs $50 \%$; $\mathrm{p}<0,05)$. Assim, apesar das limitações metodológicas do estudo aqui apresentado, os resultados obtidos se mostram bem semelhantes aos da literatura: os ciclos múltiplos de corticosteróide antenatal não parecem superiores em termos de redução da morbimortalidade de prematuros.

Vale lembrar, entretanto, que no nosso país, onde a mortalidade neonatal ainda é muito elevada, a utilização de um ciclo único de corticosteróide antenatal não deve ser esquecida diante do baixo custo e aos comprovados benefícios que promove na evolução de recém-nascidos prematuros.

\section{ABSTRACT}

Purpose: to evaluate if multiple courses of antenatal corticosteroids are more effective than single ones to reduce morbidity and mortality of preterm infants.

Methods: retrospective study of 184 newborns with gestational age less than 34 weeks from a tertiary-level hospital in São Paulo from January 1988 to December 1998. The patients were divided into two groups: single course $(n=135)$ newborns whose mothers were exposed to a complete single course ( 2 doses of betamethasone or 4 doses of dexamethasone between $24 \mathrm{~h}$ and 7 days prior to delivery); multiple courses $(n=49)$ - newborns whose mothers were exposed to two or 
more complete courses. The primary clinical outcomes for the two groups were: frequency of respiratory distress syndrome $(R D S)$, intra-hospital mortality and combined neonatal morbidity (including the presence of the following: RDS, periintraventricular hemorrhage, bronchopulmonary dysplasia, necrotizing enterocolitis, sepsis or intra-hospital death). Numerical data were compared by Student's t test or MannWhitney test and categorical data by $\chi^{2}$ or Fisher exact test, with the odds ratio and its confidence interval.

Results: there were no differences between the groups that received single or multiple courses of antenatal corticosteroids in regard to the occurrence of RDS (single course: $22 \%$ and multiple course: $18 \%$ ), intra-hospital mortality (single course $18 \%$ and multiple 12\%) and combined neonatal morbidity (single course $62 \%$ and multiple $63 \%$ ).

Conclusions: multiple courses of antenatal corticosteroids did not reduce the morbidity and mortality of preterm infants. This study emphasizes the present guidelines that recommend the use of one single course of corticosteroid for fetal maturation in pregnant women at risk for preterm delivery.

KEY WORDS: Antenatal corticosteroid. Preterm infant. Fetal maturation.

\section{Referências}

1. Liggins GC, Howie RN. A controlled trial of antepartum glucocorticoid treatment for prevention of the respiratory distress syndrome in premature infants. Pediatrics 1972; 50:515-25.

2. Crowley P, Chalmers I, Keirse MJ. The effects of corticosteroid administration before preterm delivery: an overview of the evidence from controlled trials. Br J Obstet Gynaecol 1990; 97:11-25.

3. National Institutes of Health Consensus Development Panel on the effect of corticosteroids for fetal maturation on perinatal outcomes. JAMA 1995; 273:413-8.

4. Ikegami M, Jobe AH, Newnham J, Polk DH, Willet KE, Sly P. Repetitive prenatal glucocorticoids improve lung function and decrease growth in preterm lambs. Am J Respir Crit Care Med 1997; 156:178-84.

5. Matthews SG. Antenatal glucocorticoids and programming of the developing CNS. Pediatr Res 2000; 47:291-300.

6. Huang WL, Beazley LD, Quinlivan JA, Evans SF, Newnham JP, Dunlop SA. Effect of corticosteroids on brain growth in fetal sheep. Obstet Gynecol 1999; 94:213-8.

7. Jobe AH, Wada N, Berry LM, Ikegami M, Ervin MG. Single and repetitive maternal glucocorticoid exposures reduce fetal growth in sheep. Am J Obstet Gynecol 1998; 178:880-5.

8. French NP, Hagan R, Evans SF, Godfrey M, Newnham JP. Repeated antenatal corticosteroids: size at birth and subsequent development. Am J Obstet Gynecol 1999; 180:114-21.

9. Banks BA, Cnaan A, Morgan MA, et al. Multiple courses of antenatal corticosteroids and outcome of premature neonates. Am J Obstet Gynecol 1999; 181:709-17.
10.Vermillion ST, Soper DE, Chasedunn-Roark J. Neonatal sepsis after betamethasone administration to patients with preterm premature rupture of membranes. Am J Obstet Gynecol 1999; 181:320-7.

11.Guinn DA, Atkinson MW, Sullivan L, et al. Single vs weekly courses of antental corticosteroids for women at risk of preterm delivery: a randomized controlled trial. JAMA 2001; 286:1581-7.

12.Dubowitz LM, Dubowitz V, Goldberg C. Clinical assessment of gestational age in the newborn infant. J Pediatr 1970; 77:1-10.

13.Ballard JL, Khoury JC, Wedig K, Wang L, EilersWalsman BL, Lipp R. New Ballard Score, expanded to include extremely premature infants. J Pediatr 1991; 119:417-23.

14.Battaglia FC, Lubchenco LO. A practical classification of newborn infants by weight and gestacional age. J Pediatr 1967; 71:159-63.

15.Papile LA, Burstein J, Burstein R, Koffler H, Albuquerque $\mathrm{N}$. Incidence and evolution of subependymal and intraventricular hemorrhage: a study of infants with birth weights less than 1,500gm. J Pediatr 1978; 92:529-34.

16.Walsh MC, Kliegman RM. Necrotizing enterocolitis: treatment based on staging criteria. Pediatr Clin North Am 1986; 33:179-201.

17. Antenatal corticosteroids revisited: repeated courses. NIH Consensus Statement [online] 2000 Aug 17-18; 17:1-10. Available from: URL: http:// consensus.nih.gov/cons/112/112_statement htm.

18.Agresti A. Categorical data analysis. $1^{\text {st }}$ ed. New York: John Willey and Sons; 1990.

19.Spiegel MR. Estatística. $2^{\mathrm{a}}$ ed. São Paulo: McGraw Hill; 1985.

20.Siegel S. Estadistica no parametrica. $1^{\mathrm{a}}$ ed. Mexico: Trillas; 1975.

21.Smith GN, Kingdom JC, Penning DH, Matthews SG. Antenatal corticosteroids: is more better? Lancet 2000; 355:251-2.

22. Pratt L, Waschbusch L, Ladd W, Gangnon R, Hendricks SK. Multiple vs. single betamethasone therapy: neonatal and maternal effects. J Reprod Med 1999; 44:257-64.

23.Abbasi S, Hirsch D, Davis J, et al. Effect of single versus multiple courses of antenatal corticosteroids on maternal and neonatal outcome. Am J Obstet Gynecol 2000; 182:1243-9.

24.Banks BA, Macones G, Cnaan A, Merrill JD, Ballard PL, Ballard RA. Multiple courses of antenatal corticosteroids are associated with early severe lung disease in preterm neonates. J Perinatol 2002; 22:101-7.

25.Le Cras TD, Markham NE, Morris KG, Ahrens CR, McMurtry IF, Abman SH. Neonatal dexamethasone treatment increases the risk for pulmonary hypertension in adult rats. Am J Physiol Lung Cell Mol Physiol 2000; 278:L822-9.

26.Jazayeri A, Gavrila D, Sincich T. Repeated antenatal corticosteroid treatments. Do they reduce neonatal morbidity? J Reprod Med 2001; 46:788-90.

Recebido em: 9/9/2002 Aceito com modificações em: 30/9/2002 